\title{
STABILITY, INSTABILITY IN DELAY EQUATIONS MODELING HUMAN RESPIRATION
}

\author{
By \\ Kenneth L. Cooke \\ and \\ Janos Turi
}

IMA Preprint Series \# 925

February 1992 


\title{
Stability, Instability in Delay Equations Modeling Human Respiration
}

\author{
Kenneth L. Cooke* \\ Depatment of Mathematics \\ Pomona College \\ Claremont, CA 91711
}

\author{
Janos Turi* \\ Programs in Mathematical Sciences \\ University of Texas at Dallas \\ Richardson, TX 75083
}

\begin{abstract}
A system of delay equations describing a simple model of the respiratory control mechanism in humans is considered and conditions guaranteeing stability, instability of steady-state equilibrium solutions of that system are presented.
\end{abstract}

\section{Introduction}

Understanding the mechanism producing unstable patterns of ventilation has potential significance for the prevention and treatment of various forms of irregularities in human respiration (i.e., periodic breathing, sleep apnea, SIDS, ... etc). Recent modeling studies have demonstrated that it may be beneficial to consider the above process as a manifestation of feedback induced instabilities in the respiratory system (see e.g. [9], [3], [2], [10], and the references therein). In [9] a general mathematical model to study the process leading to periodic breathing is outlined. This model can be described as a set of nonlinear parameter dependent delay differential equations with multiple circulatory transport delays. In view of the previous discussion it seems to be necessary to perform a nonlinear stability analysis, or at least a bifurcation analysis, on the proposed model equations in order to validate them for the intended purposes. (Note that in [9] the analysis is restricted to studying linearized

${ }^{*}$ Parts of this research were carried out while the authors were visitors at the Institute for Mathematics and its Applications (IMA) and were supported by IMA with funds provided by the National Science Foundation 
system response for harmonic excitations.) On the other hand, due to the complexity of the delay systems, i.e., relatively large number of states and system parameters in it, and the presence of multiple (presumably noncommensurate) delays, the only feasible way to carry out such a study is by computational means. The development and justification of computational methods to investigate stability and instability of trajectories corresponding to complex systems, such as the one in [9], is greatly aided by the availability of analytical results obtained on similar models with simpler structure.

The aim of this paper is to present a stability analysis for a simplified model of the respiratory system. (Related computational issues will be discussed in a forthcoming publication.)

In Section 2 we study modeling issues and formulate our simplified model for the respiratory system. The stability results are contained in Section 3.

\section{Model Equations}

In this section we provide a brief discussion on respiratory models to motivate the type of equations we study in Section 3.

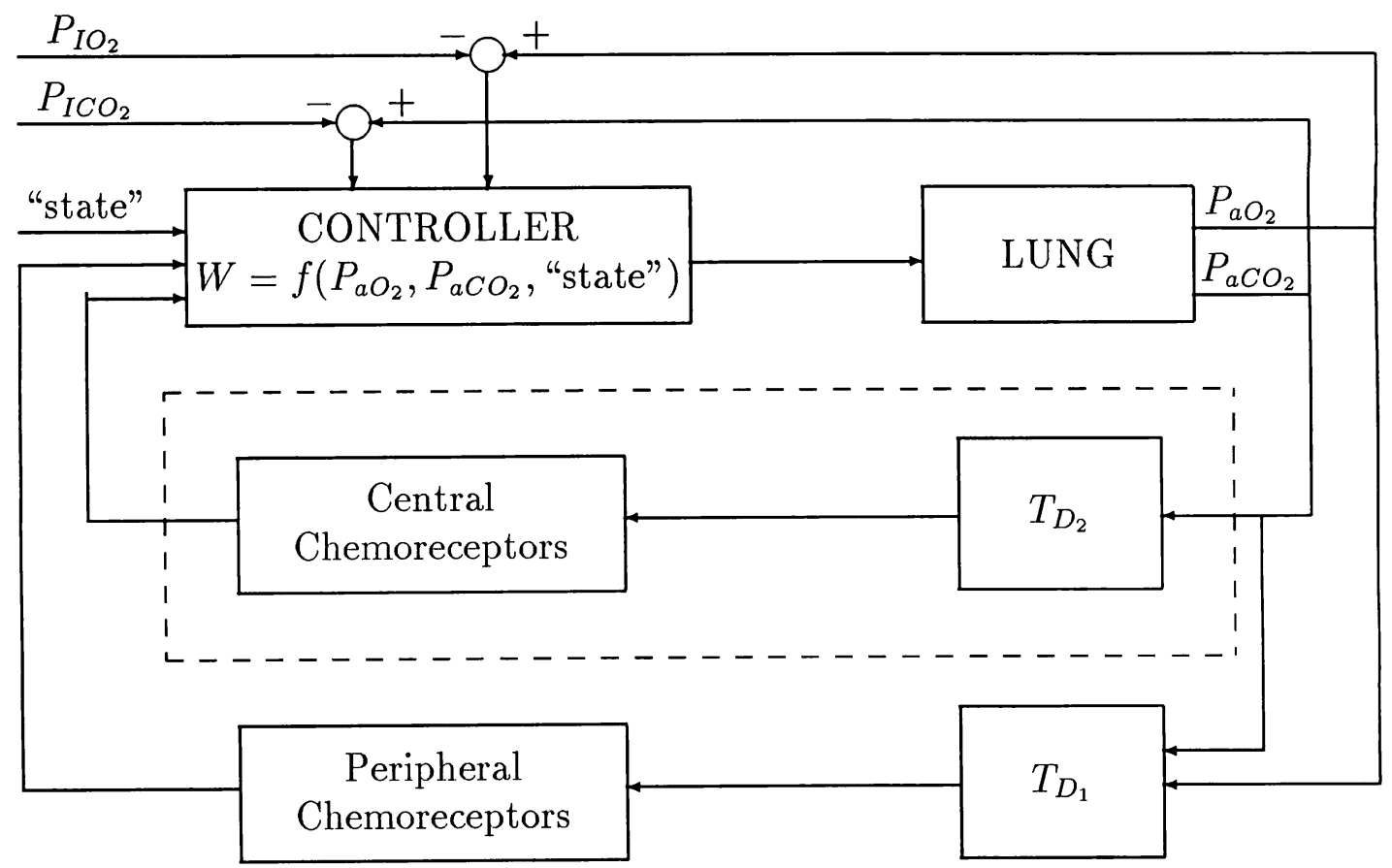

Figure 1: Block diagram of respiratory control system.

An overview of the respiratory system is shown in Figure 1. The controller responds to inputs from the central and peripheral chemoreceptors which respond to changes in $\mathrm{CO}_{2}$ 
and $\mathrm{O}_{2}$ concentrations. The input-output relationship of the controller is also influenced by changes in the state (i.e., sleep, wakefulness.) The controller gain is ventilation $(W)$, and $W$ together with the inspired $\mathrm{CO}_{2}$ and $\mathrm{O}_{2}$ concentrations (i.e., $\mathrm{PICO}_{2}$ and $\mathrm{PIO}_{2}$ ) influence the behavior of the controlled system (i.e., lung (plant)-controller closed loop, see Figure 1). The plant equations are balance equations for $O_{2}$ and $C O_{2}$ concentrations. $T_{D_{1}}$ and $T_{D_{2}}$ represent the transport delays between the lung and the peripheral and central chemoreceptors, respectively.

Khoo at al. [9] employed a model of the ventilatory system similar to the one outlined in Figure 1. The conclusion in [9] is that the ventilatory oscillations are predominantly mediated by the peripheral chemoreceptors. In this paper we concentrate on the effects due to the peripheral chemoreceptors (i.e., Figure 1 with the units inside the dotted area omitted) in the control system. Note that a similar study was carried out in [11] except that there the dependence on the $\mathrm{O}_{2}$ concentration was not included.

We consider the system of nonlinear delay equations

$$
\begin{aligned}
& \frac{d \tilde{x}}{d t}=p-\alpha W(\tilde{x}(t-\tau), \tilde{y}(t-\tau))\left(\tilde{x}(t)-x_{I}\right) \\
& \frac{d \tilde{y}}{d t}=-\sigma+\beta W(\tilde{x}(t-\tau), \tilde{y}(t-\tau))\left(y_{I}-\tilde{y}(t)\right)
\end{aligned}
$$

where $\tilde{x}(\cdot)$ and $\tilde{y}(\cdot)$ denote arterial $\mathrm{CO}_{2}$ and $\mathrm{O}_{2}$ concentrations, respectively; $W(\cdot, \cdot)$ is the ventilation function; $\tau>0$ is the transport delay (i.e., $\tau=T_{D_{1}}$ on Figure 1); $x_{I}$ and $y_{I}$ are inspired $\mathrm{CO}_{2}$ and $\mathrm{O}_{2}$ concentrations; $p$ is the $\mathrm{CO}_{2}$ production rate, $\sigma$ is the $\mathrm{O}_{2}$ consumption rate, and $\alpha, \beta$ are positive constants.

We now transform system $(2.1)$ to a form more convenient for the stability analysis in the next section by introducing

$$
x(t)=a\left(\tilde{x}(t)-x_{I}\right), \quad y(t)=b\left(y_{I}-\tilde{y}(t)\right),
$$

where $a$ and $b$ are constants to be determined. Simple calculations yield

$$
\begin{aligned}
& \frac{d x}{d t}=a \frac{d \tilde{x}(t)}{d t}=a p-a \alpha W\left(x_{I}+\frac{1}{a} x(t-\tau), y_{I}-\frac{1}{b} y(t-\tau)\right) \frac{x(t)}{a} \\
& \frac{d y}{d t}=-b \frac{d \tilde{y}(t)}{d t}=b \sigma-b \beta W\left(x_{I}+\frac{1}{a} x(t-\tau), y_{I}-\frac{1}{b} y(t-\tau)\right) \frac{y(t)}{b}
\end{aligned}
$$

Choosing the constants $a$ and $b$ as

$$
a=\frac{1}{p}, \quad b=\frac{1}{\sigma},
$$

we obtain the "scaled" equations

$$
\begin{aligned}
& \frac{d x}{d t}=1-\alpha V(x(t-\tau), y(t-\tau)) x(t) \\
& \frac{d y}{d t}=1-\beta V(x(t-\tau), y(t-\tau)) y(t)
\end{aligned}
$$


where the function $V(\cdot, \cdot)$ is defined as

$$
V(x, y)=W\left(x_{I}+p x, y_{I}-\sigma y\right)
$$

\section{$3 \quad$ Stability Analysis}

We now consider the system in scaled coordinates, (i.e. (2.3)) and investigate the questions of existence, uniqueness, and stability of equilibria. It appears to be biologically realistic (see e.g. [9]) to assume that $W(u, v)$ is increasing as a function of $u$ and decreasing as a function of $v$, for $u>x_{I}$ and $v<y_{I}$. We therefore make the following assumption (see also the defining equation (2.4)):

$$
\begin{aligned}
& V(x, y) \text { is a differentiable function, } V(0,0)=0, \text { and } \\
& \frac{\partial V(x, y)}{\partial x}>0, \frac{\partial V(x, y)}{\partial y}>0, x>0, y>0
\end{aligned}
$$

Remark 3.1 The region $x>0, y>0$ corresponds to $\tilde{x}>x_{I}, \tilde{y}<y_{I}$ in the original variables.

Theorem 3.2 Assume hypothesis (H). Then there is a unique positive equilibrium of system (2.3).

Proof: If there is an equlibirium $\bar{x}, \bar{y}$, we see from $(2.3)$ that $\bar{x} \neq 0, \bar{y} \neq 0$, and $\bar{x}=\beta \bar{y} / \alpha$. Therefore

$$
\beta V\left(\frac{\beta \bar{y}}{\alpha}, \bar{y}\right)=\frac{1}{\bar{y}}
$$

Since $V(0,0)=0$ and $V\left(\frac{\beta \bar{y}}{\alpha}, \bar{y}\right)$ is increasing in $\bar{y}$, there is a unique positive solution $\bar{y}$ of (3.1). With this $\bar{y}$, define $\bar{x}=\beta \bar{y} / \alpha$, and we have

$$
\alpha V(\bar{x}, \bar{y})=\alpha V\left(\frac{\beta \bar{y}}{\alpha}, \bar{y}\right)=\frac{\alpha}{\beta \bar{y}}=\frac{1}{\bar{x}}
$$

Hence $\alpha \bar{x} V(\bar{x}, \bar{y})=1=\beta \bar{y} V(\bar{x}, \bar{y})$ so $(\bar{x}, \bar{y})$ is an equilibrium.

Example 3.3 In the paper of Mackey and Glass [11], the function $V(x)$ was taken, as example, to be of the form $V(x)=V_{m} x^{n} /\left(\Theta^{n}+x^{n}\right)$, where $V_{m}$ and $\Theta$ are positive constants and $n$ is a positive integer. Analogously, we may consider the radially symmetric function

$$
V(r) \equiv V(x, y)=\frac{V_{m} r^{n}}{\Theta^{n}+r^{n}}, \quad r=\sqrt{x^{2}+y^{2}}
$$

which satisfies $\partial V / \partial x>0, \partial V / \partial y>0$ when $x>0, y>0$. In the case $n=1$, we can explicitly compute the equilibrium $\bar{r}=\sqrt{\bar{x}^{2}+\bar{y}^{2}}$. Since $\bar{x}=\beta \bar{y} / \alpha$,

$$
\bar{r}^{2}=\left(\frac{\beta \bar{y}}{\alpha}\right)^{2}+\bar{y}^{2}=k^{2} \bar{y}^{2}, \quad k^{2}=\frac{\beta^{2}+\alpha^{2}}{\alpha^{2}} .
$$


Equation (3.1) becomes

$$
\frac{\beta V_{m} \bar{r}}{\Theta+\bar{r}}=\frac{k}{\bar{r}}
$$

and therefore

$$
\beta V_{m} \bar{r}^{2}-k \bar{r}-k \Theta=0 .
$$

Solving (3.3) we obtain

$$
\bar{r}=\frac{1}{2} \delta\left\{1+\sqrt{1+\frac{4 \Theta}{\delta}}\right\}
$$

where $\delta=\frac{k}{\beta V_{m}}$.

We now investigate asymptotic stability of the equlibrium of system (2.3), under hypothesis $(\mathrm{H})$. By letting $\xi(t)=x(t)-\bar{x}, \eta(t)=y(t)-\bar{y}$ in (2.3), and then removing the nonlinear terms, we obtain the linear variational system

$$
\begin{aligned}
& \frac{d \xi}{d t}=-\alpha \bar{V} \xi(t)-\alpha \bar{x} \bar{V}_{x} \xi(t-\tau)-\alpha \bar{x} \bar{V}_{y} \eta(t-\tau) \\
& \frac{d \eta}{d t}=-\beta \bar{V} \eta(t)-\beta \bar{y} \bar{V}_{x} \xi(t-\tau)-\beta \bar{y} \bar{V}_{y} \eta(t-\tau)
\end{aligned}
$$

where $\bar{V}=V(\bar{x}, \bar{y}), \bar{V}_{x}=V_{x}(\bar{x}, \bar{y})$, and $\bar{V}_{y}=V_{y}(\bar{x}, \bar{y})$. This may also be written in the form

$$
\frac{d}{d t}\left(\begin{array}{c}
\xi(t) \\
\eta(t)
\end{array}\right)+A\left(\begin{array}{c}
\xi(t) \\
\eta(t)
\end{array}\right)+B\left(\begin{array}{c}
\xi(t-\tau) \\
\eta(t-\tau)
\end{array}\right)=\left(\begin{array}{l}
0 \\
0
\end{array}\right),
$$

where

$$
A=\left(\begin{array}{cc}
\alpha \bar{V} & 0 \\
0 & \beta \bar{V}
\end{array}\right), B=\left(\begin{array}{cc}
\alpha \bar{x} \bar{V}_{x} & \alpha \bar{x} \bar{V}_{y} \\
\beta \bar{y} \bar{V}_{x} & \beta \bar{y} \bar{V}_{y}
\end{array}\right) .
$$

The associated characteristic equation ( see [1]) is

$$
\operatorname{det}\left(\lambda I+A+B e^{-\tau \lambda}\right)=0 .
$$

Let us first consider the case $\tau=0$, for which (3.5) reduces to

$$
\lambda^{2}+\left(\alpha \bar{V}+\alpha \bar{x} \bar{V}_{x}+\beta \bar{V}+\beta \bar{y} \bar{V}_{y}\right) \lambda+\alpha \beta\left(\bar{V}^{2}+\bar{x} \bar{V} \bar{V}_{x}+\bar{y} \bar{V} \bar{V}_{y}\right)=0 .
$$

Because of the hypothesis $(\mathrm{H})$, the coefficients in this equation are positive, and therefore the roots have negative real parts. Consequently, we have proved the following result.

Theorem 3.4 If (H) holds and $\tau=0$, the positive equilibrium $(\bar{x}, \bar{y})$ is asymptotically stable. 
Now suppose that $\tau>0$. Equation (3.5) then has the form

$$
P(\lambda)+Q(\lambda) e^{-\tau \lambda}=0
$$

where

$$
P(\lambda)=\lambda^{2}+(\alpha+\beta) \bar{V} \lambda+\alpha \beta \bar{V}^{2}
$$

and

$$
Q(\lambda)=\left(\alpha \bar{x} \bar{V}_{x}+\beta \bar{y} \bar{V}_{y}\right) \lambda+\alpha \beta \bar{V}\left(\bar{x} \bar{V}_{x}+\bar{y} \bar{V}_{y}\right) .
$$

It is well-known that a necessary and sufficient condition for asymptotic stability of the equilibrium is that all roots of the charasteristic equation have negative real parts. Therefore, much effort has been devoted to searching for conditions on the polynomials $P$ and $Q$ that will imply that all roots have negative real parts. References to some of this literature may be found for example in [1], [8], [12], [4], [5]. Since $P+Q e^{-\tau \lambda}$ is an exponential polynomial, these conditions are somewhat difficult to express in a way that is useful in applications, and this is even more so for exponential polynomials coming from equations with more than one delay.

At the end of this section, we comment further on equations (3.6)- (3.8), but first we construct a special case wich is more easily handled. The special case is when $\alpha=\beta$. Equation (3.6) then reduces to

$$
(\lambda+\alpha \bar{V})\left[\lambda+\alpha \bar{V}+\alpha\left(\bar{x} \bar{V}_{x}+\bar{y} \bar{V}_{y}\right) e^{-\tau \lambda}\right]=0,
$$

and consequently we only need to discuss the location of roots of the equation

$$
\lambda+a+b e^{-\tau \lambda}=0, \quad a=\alpha \bar{V}, \quad b=\alpha\left(\bar{x} \bar{V}_{x}+\bar{y} \bar{V}_{y}\right) .
$$

The exact region of stability for (3.9) is the region in the $(a, b)$-plane bounded on the left by the line $a+b=0, a \tau \geq-1$, and with upper boundary given by the equation

$$
\tau\left(b^{2}-a^{2}\right)^{1 / 2}=\arccos (-a / b), \quad a \tau \geq-1 .
$$

([7], page 149). In the present case since we have $a>0, b>0$, the lower boundary is simply the positive $a$-axis. Moreover, the region of stability for all delays $\tau$ is simply $b<a$. The following result is therefore true.

Theorem 3.5 If (H) holds and $\alpha=\beta$, the equilibrium $(\bar{x}, \bar{y})$ is asymptotically stable if, and only if, the parameter pair $(a, b)$ lies in the region in the first quadrant with $a>0, b>0$, and upper boundary $\tau\left(b^{2}-a^{2}\right)^{1 / 2}=\arccos (-a / b)$, where $a=\alpha \bar{V}, b=\alpha\left(\bar{x} \bar{V}_{x}+\bar{y} \bar{V}_{y}\right)$. The equilibrium is stable for all $\tau \geq 0$ if and only if $b<a$.

If the parameter pair moves across the upper boundary, there is generally a Hopf bifurcation with emergence of a nontrivial periodic solution. 
Example 3.6 For the situation described in Example 3.3, we have

$$
a=\frac{\alpha V_{m} \bar{r}^{n}}{\Theta^{n}+\bar{r}^{n}}, \quad b=\frac{\alpha n V_{m} \theta^{n} \bar{r}^{n}}{\left(\Theta^{n}+\bar{r}^{n}\right)^{2}} .
$$

The condition for stability for all delays is $b<a$, which is $\bar{r}^{n}>(n-1) \Theta^{n}$. For $n=1$, this is clearly true. For $n>1$, since $k=\sqrt{2}$ we have $g \bar{r}^{n+1}=\bar{r}^{n}+\theta^{n}$ where $g=\alpha V_{m} / \sqrt{2}$, hence $\bar{r} \rightarrow 0$ as $g \rightarrow \infty$. Therefore the condition $\bar{r}>(n-1) \theta^{n}$ fails if $\alpha V_{m}$ is large, which corresponds to overly strong gain in the regulation.

We now return to a discussion of the general case, when $\alpha \neq \beta$, and to the equation (3.6). For equations with quadratic function $P$ and linear function $Q$, a survey of what is known about stability in various cases is given in [4]. The recent book [12] provides a method for determining stability for more general problems, including problems with several delays, and is based on determining roots of certain real functions. Papers [6] and [5] show that if $\tau$ is regarded as a parameter, then as $\tau$ increases there may be a sequence of switches between stability and instability. For our purposes here, we construct the function

$$
\begin{aligned}
F(\omega) & =|P(i \omega)|^{2}-|Q(i \omega)|^{2} \\
& =\omega^{4}+\left[\left(\alpha^{2}+\beta^{2}\right) \bar{V}^{2}-\left(\alpha \bar{x} \bar{V}_{x}+\beta \bar{y} \bar{V}_{y}\right)^{2}\right] \omega^{2}+\alpha^{2} \beta^{2} \bar{V}^{2}\left[\bar{V}-\left(\bar{x} \bar{V}_{x}+\bar{y} \bar{V}_{y}\right)^{2}\right]
\end{aligned}
$$

Since we have stability for $\tau=0$, by Theorem 3.4, the following result follows from [5], Theorem 1.

Theorem 3.7 Assume hypothesis (H). Then:

(i) If $F(\omega)=0$ has no positive roots, then the equilibrium $(\bar{x}, \bar{y})$ is asymptotically stable for all $\tau \geq 0$.

(ii) If $F(\omega)=0$ has at least one positive root $\omega$, and each positive root is simple, then as $\tau$ increases, stability switches may occur and there is a positive $\tau^{*}$ such that the equilibrium is unstable for all $\tau>\tau^{*}$.

We observe that if $\bar{V}<\bar{x} \bar{V}_{x}+\bar{y} \bar{V}_{y}$, then $F(0)$ is negative and there is a positive root of $F(\omega)=0$, and destabilization will occur for large $\tau$. More precise conditions for the existence of a positive root of $F(\omega)=0$ may be obtained similarly as in ([6], Section 5).

\section{References}

[1] R. Bellman and K. L. Cooke, "Differential-Difference Equations", Academic Press, New York, 1963.

[2] G. P. Brady and E. M. McCann, Control of ventilation in subsequent siblings of victims of sudden infant death syndrome, J. Pediatrics 106 (1985), 212-217. 
[3] N. S. Cherniack and G. S. Longobardo, Abnormalities in respiratory rhythm, "In: Handbook of Physiology: The Respiratory System", Vol. 2, 729-750, American Physiological Society, 1986.

[4] J. Chuma and P. van den Driessche, A general second-order transcendental equation, Appl. Math. Notes 5 (1980), 85-96.

[5] K. L. Cooke and P. van den Driessche, On zeroes of some transcendental equations, Funkcialaj Ekvacioj 29 (1986), 77-90.

[6] K. L. Cooke and Z. Grossman, Discrete delay, distributed delay and stability switches, J. Math. Anal. Appl. 86 (1982), 592-627.

[7] L. E El'sgol'ts and S. B. Norkin, "Introduction to the Theory and Application of Differential Equations with Deviating Arguments", Academic Press, New York, 1973.

[8] J. K. Hale, "Theory of Functional Differential Equations", Springer-Verlag, New York, 1977.

[9] M. C. K. Khoo, R. E. Kronauer, K. P. Strohl and A. S. Slutsky, Factors inducing periodic breathing in humans: a general model, J. Appl. Physiol. 53 (1982), 644-659.

[10] G. S. Longobardo, B. Gothe, M. D. Goldman and N. S. Cherniack, Sleep apnea considered as a control system instability, Resp. Physiol. 50 (1982), 311-333.

[11] M. C. Mackey and L. Glass, Oscillation and chaos in physiological control systems, Science 197 (1977), 287-289.

[12] G. Stépán, "Retarded Dynamical Systems: Stability and Characteristic Functions", Pitman Research Notes in Math. No. 210, Longman Scientific \& Technical, Harlow, U.K. 1989. 
839 Oscar P. Bruno and Fernando Reitich, Numerical solution of diffraction problems: a method of variation of boundaries

Oscar P. Bruno and Fernando Reitich, Solution of a boundary value problem for Helmholtz equation via variation of the boundary into the complex domain

Victor A. Galaktionov and Juan L. Vazquez, Asymptotic behaviour for an equation of superslow diffusion. The Cauchy problem

Josephus Hulshof and Juan Luis Vazquez, The Dipole solution for the porous medium equation in several space dimensions

Shoshana Kamin and Juan Luis Vazquez, The propagation of turbulent bursts

Miguel Escobedo, Juan Luis Vazquez and Enrike Zuazua, Source-type solutions and asymptotic behaviour for a diffusion-convection equation

Marco Biroli and Umberto Mosco, Discontinuous media and Dirichlet forms of diffusion type

Stathis Filippas and Jong-Shenq Guo, Quenching profiles for one-dimensional semilinear heat equations

H. Scott Dumas, A Nekhoroshev-like theory of classical particle channeling in perfect crystals

R. Natalini and A. Tesei, On a class of perturbed conservation laws

Paul K. Newton and Shinya Watanabe, The geometry of nonlinear Schrödinger standing waves

S.S. Sritharan, On the nonsmooth verification technique for the dynamic programming of viscous flow

Mario Taboada and Yuncheng You, Global attractor, inertial manifolds and stabilization of nonlinear damped beam equations

Shigeru Sakaguchi, Critical points of solutions to the obstacle problem in the plane

F. Abergel, D. Hilhorst and F. Issard-Roch, On a dissolution-growth problem with surface tension in the neighborhood of a stationary solution

Erasmus Langer, Numerical simulation of MOS transistors

Haïm Brezis and Shoshana Kamin, Sublinear elliptic equations in $\mathbf{R}^{n}$

Johannes C.C. Nitsche, Boundary value problems for variational integrals involving surface curvatures

Chao-Nien Chen, Multiple solutions for a semilinear elliptic equation on $\mathbf{R}^{N}$ with nonlinear dependence on the gradient

D. Brochet, X. Chen and D. Hilhorst, Finite dimensional exponential atttractor for the phase field model

Joseph D. Fehribach, Mullins-Sekerka stability analysis for melting-freezing waves in helium-4

Walter Schempp, Quantum holography and neurocomputer architectures

D.V. Anosov, An introduction to Hilbert's 21st problem

Herbert E Huppert and M Grae Worster, Vigorous motions in magma chambers and lava lakes

Robert L. Pego and Michael I. Weinstein, A class of eigenvalue problems, with applications to instability of solitary waves

Mahmoud Affouf, Numerical study of a singular system of conservation laws arising in enhanced oil reservoirs

Darin Beigie, Anthony Leonard and Stephen Wiggins, The dynamics associated with the chaotic tangles of two dimensional quasiperiodic vector fields: theory and applications

Gui-Qiang Chen and Tai-Ping Liu, Zero relaxation and dissipation limits for hyperbolic conservation laws

Gui-Qiang Chen and Jian-Guo Liu, Convergence of second-order schemes for isentropic gas dynamics

Aleksander M. Simon and Zbigniew J. Grzywna, On the Larché-Cahn theory for stress-induced diffusion

Jerzy Luczka, Adam Gadomski and Zbigniew J. Grzywna, Growth driven by diffusion

Mitchell Luskin and Tsorng-Whay Pan, Nonplanar shear flows for nonaligning nematic liquid crystals

Mahmoud Affouf, Unique global solutions of initial-boundary value problems for thermodynamic phase transitions

Richard A. Brualdi and Keith L. Chavey, Rectangular $L$-matrices

Xinfu Chen, Avner Friedman and Bei Hu, The thermistor problem with zero-one conductivity II

Raoul LePage, Controlling a diffusion toward a large goal and the Kelly principle

Raoul LePage, Controlling for optimum growth with time dependent returns

Marc Hallin and Madan L. Puri, Rank tests for time series analysis a survey

V.A. Solonnikov, Solvability of an evolution problem of thermocapillary convection in an infinite time interval

Horia I. Ene and Bogdan Vernescu, Viscosity dependent behaviour of viscoelastic porous media

Kaushik Bhattacharya, Self-accommodation in martensite

D. Lewis, T. Ratiu, J.C. Simo and J.E. Marsden, The heavy top: a geometric treatment

Leonid V. Kalachev, Some applications of asymptotic methods in semiconductor device modeling

David C. Dobson, Phase reconstruction via nonlinear least-squares

Patricio Aviles and Yoshikazu Giga, Minimal currents, geodesics and relaxation of variational integrals on mappings of bounded variation 
Charles R. Johnson and Michael Lundquist, Operator matrices with chordal inverse patterns

B.J. Bayly, Infinitely conducting dynamos and other horrible eigenproblems

Charles M. Elliott and Stefan Luckhaus, 'A generalised diffusion equation for phase separation of a multi-compon mixture with interfacial free energy'

888

889

890

891

892

893

894

895

896

898

899

900

901

902

903

904

905

906

907

908

909

910

911

912

913

914

915

916

917

918

919
Christian Schmeiser and Andreas Unterreiter, The derivation of analytic device models by asymptotic methods

LeRoy B. Beasley and Norman J. Pullman, Linear operators that strongly preserve the index of imprimitivity

Jerry Donato, The Boltzmann equation with lie and cartan

Thomas R. Hoffend Jr., Peter Smereka and Roger J. Anderson, A method for resolving the laser induced local heating of moving magneto-optical recording media

E.G. Kalnins, Willard Miller, Jr. and Sanchita Mukherjee, Models of $q$-algebra representations: the group of plane motions

T.R. Hoffend Jr. and R.K. Kaul, Relativistic theory of superpotentials for a nonhomogeneous, spatially isotropic medium

Reinhold von Schwerin, Two metal deposition on a microdisk electrode

Vladimir I. Oliker and Nina N. Uraltseva, Evolution of nonparametric surfaces with speed depending on curvature, III. Some remarks on mean curvature and anisotropic flows

Wayne Barrett, Charles R. Johnson, Raphael Loewy and Tamir Shalom, Rank incrementation via diagonal perturbations

Mingxiang Chen, Xu-Yan Chen and Jack K. Hale, Structural stability for time-periodic one-dimensional parabolic equations

Hong-Ming Yin, Global solutions of Maxwell's equations in an electromagnetic field with the temperaturedependent electrical conductivity

Robert Grone, Russell Merris and William Watkins, Laplacian unimodular equivalence of graphs

Miroslav Fiedler, Structure-ranks of matrices

Miroslav Fiedler, An estimate for the nonstochastic eigenvalues of doubly stochastic matrices

Miroslav Fiedler, Remarks on eigenvalues of Hankel matrices

Charles R. Johnson, D.D. Olesky, Michael Tsatsomeros and P. van den Driessche, Spectra with positive elementary symmetric functions

Pierre-Alain Gremaud, Thermal contraction as a free boundary problem

K.L. Cooke, Janos Turi and Gregg Turner, Stabilization of hybrid systems in the presence of feedback delays

Robert P. Gilbert and Yongzhi Xu, A numerical transmutation approach for underwater sound propagation

LeRoy B. Beasley, Richard A. Brualdi and Bryan L. Shader, Combinatorial orthogonality

Richard A. Brualdi and Bryan L. Shader, Strong hall matrices

Håkan Wennerström and David M. Anderson, Difference versus Gaussian curvature energies; monolayer versus bilayer curvature energies applications to vesicle stability

Shmuel Friedland, Eigenvalues of almost skew symmetric matrices and tournament matrices

Avner Friedman, Bei Hu and J.L. Velazquez, A Free Boundary Problem Modeling Loop Dislocations in Crystals

Ezio Venturino, The Influence of Diseases on Lotka-Volterra Systems

Steve Kirkland and Bryan L. Shader, On Multipartite Tournament Matrices with Constant Team Size

Richard A. Brualdi and Jennifer J.Q. Massey, More on Structure-Ranks of Matrices

Douglas B. Meade, Qualitative Analysis of an Epidemic Model with Directed Dispersion

Kazuo Murota, Mixed Matrices Irreducibility and Decomposition

Richard A. Brualdi and Jennifer J.Q. Massey, Some Applications of Elementary Linear Algebra in Combinations

Carl D. Meyer, Sensitivity of Markov Chains

Hong-Ming Yin, Weak and Classical Solutions of Some Nonlinear Volterra Integrodifferential Equations

B. Leinkuhler and A. Ruehli, Exploiting Symmetry and Regularity in Waveform Relaxation Convergence Estimation

Xinfu Chen and Charles M. Elliott, Asymptotics for a Parabolic Double Obstacle Problem

Yongzhi Xu and Yi Yan, An Approximate Boundary Integral Method for Acoustic Scattering in Shallow Oceans

Yongzhi Xu and Yi Yan, Source Localization Processing in Perturbed Waveguides

Kenneth L. Cooke and Janos Turi, Stability, Instability in Delay Equations Modeling Human Respiration

F. Bethuel, H. Brezis, B.D. Coleman and F. Hélein, Bifurcation Analysis of Minimizing Harmonic Maps Describing the Equilibrium of Nematic Phases Between Cylinders

Frank W. Elliott, Jr., Signed Random Measures: Stochastic Order and Kolmogorov Consistency Conditions

D.A. Gregory, S.J. Kirkland and B.L. Shader, Pick's Inequality and Tournaments

J.W. Demmel, N.J. Higham and R.S. Schreiber, Block $L U$ Factorization

Victor A. Galaktionov and Juan L. Vazquez, Regional Blow-Up in a Semilinear Heat Equation with Convergence to a Hamilton-Jacobi Equation

Bryan L. Shader, Convertible, Nearly Decomposable and Nearly Reducible Matrices

Dianne P. O'Leary, Iterative Methods for Finding the Stationary Vector for Markov Chains 